\title{
A randomized study of the efficacy and safety of parecoxib for the treatment of pain following total knee arthroplasty in Korean patients
}

This article was published in the following Dove Press journal: Journal of Pain Research

\section{Margaret Noyes Essex' Hee-Youn Choi ${ }^{2}$ \\ Pritha Bhadra Brown' Raymond Cheung'}

'Pfizer Inc., New York, NY, USA; ${ }^{2}$ Pfizer Pharmaceutical Korea Ltd, Seoul, South Korea
Correspondence: Margaret Noyes Essex Pfizer Inc., 235 East 42nd Street, New York, NY, USA

$\mathrm{Tel}+\mathrm{I} 2127338018$

Fax + I $64641 \mid 5967$

Email margaret.essex@pfizer.com
Purpose: Parecoxib is an injectable cyclooxygenase-2 inhibitor with proven postoperative analgesic efficacy in a variety of settings, including total knee arthroplasty (TKA). The effect of ethnicity on the efficacy of parecoxib for post-TKA pain has not been studied.

Patients and methods: This was a parallel-group, double-blind, randomized, placebocontrolled study of ethnically Korean patients aged $\geq 18$ years who had unilateral TKA. Patients who reported moderate or severe pain 6 hours after the end of postoperative opioid analgesia were randomized to receive a single intravenous dose of parecoxib sodium $40 \mathrm{mg}$ or placebo. Patients were evaluated for 24 hours postdose. The primary efficacy endpoints included time-specific pain intensity difference (PID), time-specific pain relief (PR), and time to rescue medication. The incidence and nature of adverse events (AEs) assessed safety.

Results: Of the 116 patients randomized, 58 received parecoxib and 58 placebo. Mean (SD) PID was significantly greater for parecoxib vs placebo 1 hour postdose $(0.69$ [0.67] vs 0.40 [0.59], respectively; $p<0.05$ ), and for each time point up to 24 hours. Similarly, mean (SD) PR was significantly greater for parecoxib vs placebo 1.5 hours postdose (1.63 [0.85] vs 1.07 [0.90], respectively; $p=0.001$ ), and for each time point up to 24 hours. The median time (hours:minutes) to rescue medication was significantly longer for parecoxib vs placebo $(21: 30 \mathrm{vs} 4: 08$, respectively; $p<0.001)$. Generally, fewer AEs were reported with parecoxib than placebo, and the AE profile was consistent with previous studies. These results are comparable to the findings from a similarly designed study in a Caucasian patient population.

Conclusion: Parecoxib $40 \mathrm{mg}$ significantly improved postoperative pain vs placebo in Korean patients after TKA. The efficacy and safety of parecoxib in Korean patients is similar to that seen in Caucasian patients.

Keywords: COX-2 inhibitor, parecoxib, total knee arthroplasty

\section{Introduction}

Total knee arthroplasty (TKA) is one of the most common and effective surgical procedures but can be associated with considerable postoperative pain. According to joint registries from nine different countries, Korea had the highest growth rate $(407 \%)$ in primary TKA procedures from 2001 to 2010 , with $\sim 75,434$ performed in 2010 alone. ${ }^{1}$

More than half of the patients undergoing TKA report severe postoperative pain. ${ }^{2}$ Poor management of acute postoperative pain may be a risk factor for developing chronic pain. ${ }^{3}$ Prolonged exposure to nociceptive and inflammatory signals from the periphery may cause long-term changes in the central nervous system resulting in chronic pain. ${ }^{4}$ Thus, the goals of perioperative pain management are to treat acute pain 
and to prevent chronic pain, which contributes to increasing health care costs, slower recovery times, and patient/ caregiver burden. ${ }^{5,6}$

Opioids are the most common choice for perioperative pain; however, the current clinical recommendation is a multimodal analgesic approach intended both to efficiently manage pain and to reduce opioid use and opioid-related adverse events (AEs). ${ }^{2,5,6}$ The Korean Knee Society recommends a combination of drugs with varying mechanisms of action, such as acetaminophen, opioids, and cyclooxygenase-2 (COX-2) inhibitors. ${ }^{6}$

Parecoxib is an injectable COX-2 inhibitor with proven efficacy for postoperative pain in different surgical settings, including for gynecological surgery, ${ }^{7}$ oral surgery, ${ }^{8}$ and total hip arthroplasty or TKA. ${ }^{9-11}$ Parecoxib also has opioidsparing effects. In previous studies, parecoxib reduced opioid analgesic requirements after gynecological surgery ${ }^{12}$ and produced stronger and longer-lasting analgesia than morphine after unilateral TKA. ${ }^{11}$ Parecoxib is advantageous over nonselective nonsteroidal anti-inflammatory drugs (NSAIDs), which target both COX-1 and COX-2, in that it selectively targets the inducible COX-2 enzyme, which decreases the risk of problems associated with COX-1 inhibition, such as perioperative bleeding because of platelet aggregation inhibition $^{13}$ and gastric mucosal damage. ${ }^{14}$

Pain perception is subjective and may be influenced by a variety of factors, including ethnicity. ${ }^{15}$ Healthy volunteers who self-identified as Asian/Pacific Islander reported significantly greater acute experimental pain intensity compared with self-identified Caucasians. ${ }^{16}$ A similar study comparing self-identified non-Hispanic Caucasians with Asians yielded comparable results. ${ }^{17}$ Non-Caucasian (Asian, black, and Latino) patients with terminal and end-stage illnesses also reported more pain than Caucasian patients in another study. ${ }^{18}$ Ethnicity may also affect response to pain treatments. Following 4-week multidisciplinary treatment for chronic pain, only non-Hispanic Caucasian patients reported reduced pain severity compared with African-Americans, although both groups had improved depressive symptoms and pain-related interference. ${ }^{19}$ Conversely, African-American osteoarthritis patients reported greater benefits of over-thecounter analgesics than Caucasian osteoarthritis patients. ${ }^{20}$ The effect of ethnicity on parecoxib efficacy and safety has not been extensively studied. This study assessed the analgesic efficacy and safety of intravenous (IV) parecoxib sodium $40 \mathrm{mg}$ for postoperative pain after TKA in a Korean patient population.

\section{Materials and methods}

\section{Study design}

This study was a multicenter, parallel-group, double-blind, single-dose, placebo-controlled, randomized study conducted at four study centers in Korea from November 11, 2002 to April 8, 2003. The study was designed to replicate a clinical trial conducted in primary TKA patients in the USA. ${ }^{11}$ The appropriate Institutional Review Board at each study center approved the study (Seoul National University Hospital, Seoul; Yonsei University Medical Center, Seoul; St Mary's Hospital, Seoul; and Kyung Hee University Hospital, Seoul), and all subjects provided written informed consent before entering the study. The study was conducted in compliance with the Declaration of Helsinki and all International Conference on Harmonisation Good Clinical Practice Guidelines. This study was conducted before registration was required in a public trials registry.

\section{Patients}

Patients were male or female (nonpregnant and nonlactating) $\geq 18$ years of age who were undergoing nonemergency unilateral knee replacement surgery. Patients needed to have moderate or severe baseline pain within 6 hours after the end of patient-controlled analgesia (PCA) with morphine or meperidine, and to have discontinued continuous passive motion.

Patients were excluded from the study if they had a history of hypersensitivity to any NSAID, sulfonamide, opiate, or any analgesic used in this study. Other exclusion criteria included having a history of known alcohol, analgesic, or narcotic substance abuse within 90 days before screening, and receiving analgesics other than those required for surgery during the 6 hours preceding surgery or following administration of study medication that could confound assessment of analgesic activity. Patients were also excluded if they had been diagnosed as having or had treatment initiated for esophageal, gastric, pyloric channel, or duodenal ulceration within the 30 days before receiving study medication, or if they were experiencing significant gastrointestinal complaints as determined by the investigator.

\section{Treatment}

The day after surgery, patients were randomized by a computer-generated randomization schedule in a 1:1 ratio to receive a single, IV, double-blind dose of parecoxib sodium $40 \mathrm{mg}$ or placebo. Patients were stratified based on their baseline pain intensity (moderate or severe on a 4-point 
categorical scale where $0=$ no pain and $3=$ severe pain). PCA was used if necessary during the immediate postoperative period until 12:00 pm the first day following surgery. Study drug was administered within 6 hours after PCA ended.

Concomitant treatments including low-dose aspirin (<325 mg); chronic, stable doses of sedatives, anxiolytics, and antidepressants; ondansetron; and short-acting local lidocaine injections were allowed. Antihistamines, epidurals, intrathecal narcotics, antiemetics other than ondansetron, local or topical analgesics, and NSAIDs within 24 hours of surgery start were not permitted. Rescue medication was provided at the discretion of the study investigator.

\section{Primary endpoints}

Primary endpoints were time-specific pain intensity difference (PID), time-specific pain relief (PR), time-specific sum of PID and PR (PRID), each at time points up to 24 hours postdose, and time to rescue medication. Patients' self-rated pain intensity (on a scale from $0=$ none to $3=$ severe) and $P R$ (on a scale from $0=$ none to $4=$ complete) was assessed at the following time points: baseline (time 0 - pain intensity only); $0.25,0.5,0.75,1,1.5,2,3,4,5,6,7,8,10,12,16$, and 24 hours postdose. For each of these measurements, a larger number indicates greater improvement in PR.

\section{Secondary endpoints}

Secondary endpoints included peak pain intensity difference (PPID) and peak pain relief (PPR), both defined as the highest score achieved at any time during the evaluation period. Other secondary endpoints were the proportion of patients requiring rescue medication and the Patient's Global Evaluation of Study Medication (on a scale from 1=poor to $4=$ =xcellent), which was recorded upon treatment completion or at the time of rescue medication.

\section{Safety}

Safety was evaluated by the incidence and nature of AEs. Investigators assessed severity and whether AEs were related to study treatment. All AEs were coded and summarized by treatment group using the World Health Organization adverse reaction terminology.

\section{Statistics}

A sample size of 50 patients per treatment group was sufficient to detect a difference with at least $85 \%$ power, type 1 error at 0.05 , based on effect sizes of at least 0.63 for PID and PR at 1 and 1.5 hours. All statistical comparisons were made at the 5\% significance level. All randomized patients who received at least one dose of study medication were included in the safety analyses. The incidence of AEs between treatment groups was compared using Fisher's exact test. All efficacy analyses were done in the modified intent-to-treat population, defined as all randomized patients who received at least one dose of study medication without taking rescue medication within 1 hour postdose or missing two consecutive scheduled pain assessments in the first 2 hours.

Time-specific PID was calculated by subtracting the current pain intensity score from the baseline pain intensity score. PID, PR, PRID, PPID, and PPR were analyzed using analysis of covariance with treatment and center as factors, and baseline pain intensity as a covariate. For the time-torescue-medication analysis, patients who did not request rescue medication were assigned times censored at 24 hours. Patients who withdrew without needing rescue medication were assigned times censored at the time of study withdrawal. The median time to rescue medication, with corresponding 95\% CIs, was determined using the Kaplan-Meier product limit estimator with the adjustment given by Miller ${ }^{21}$ and the method of Simon and Lee, ${ }^{22}$ respectively. The proportion of patients requesting rescue medication was compared between treatment groups using a Cochran-Mantel-Haenszel $(\mathrm{CMH})$ test (general association), controlling for center, and did not include patients who withdrew without receiving rescue medication. The Patient's Global Evaluation of Study Medication was analyzed using the $\mathrm{CMH}$ method (modified ridits), controlling for center.

Missing pain scores were imputed using linear interpolation between observed scores on a patient-by-patient basis. A time window of \pm 5 minutes in the first 0.75 hours and of \pm 10 minutes in the remaining observation period after 0.75 hours was used in the linear interpolation. Patients who withdrew prior to the end of the study were inputted using a last-observation-carried-forward approach.

\section{Results}

\section{Patients}

The patient population (placebo, $n=58$; parecoxib, $n=58$ ) was ethnically Korean, predominantly female ( $>98 \%$ ), and had a mean age of $\sim 67$ years. Demographic and baseline characteristics were similar between the placebo and parecoxib groups, particularly with respect to baseline pain intensity, duration of surgery, and duration of anesthesia (Table 1). There was no significant difference between time from end of surgery, end of anesthesia, or end of PCA or continuous passive motion to study medication (data not shown). Approximately two-thirds of patients reported moderate baseline pain, while one-third 
reported severe pain (Table 1). All patients completed the study (Figure 1).

\section{Efficacy}

Mean (SD) PID was significantly greater in the parecoxib group compared with placebo starting at 1 hour postdose $(0.69$ [0.67] vs 0.40 [0.59], respectively; $p<0.05$ ) and for each subsequent time point throughout the 24-hour evaluation period (0.54 [0.99] vs -0.05 [0.77], respectively, at 24 hours; $p<0.001$ for all time points; Figure 2A). Mean (SD) PR was signifi-

Table I Patient baseline demographic and clinical characteristics

\begin{tabular}{lll}
\hline Characteristics & Placebo $(\mathbf{n = 5 8 )}$ & Parecoxib (n=58) \\
\hline Mean (SD) age, years & $67.6(4.96)$ & $66.2(6.65)$ \\
$\quad$ Range & $55-77$ & $46-84$ \\
Women, $\mathrm{n}(\%)$ & $58(100.0)$ & $56(97.0)$ \\
Asian, $\mathrm{n}(\%)$ & $58(100.0)$ & $58(100.0)$ \\
Mean height (SD), cm & $152.62(6.50)$ & $153.09(5.95)$ \\
$\begin{array}{l}\text { Mean weight (SD), kg } \\
\text { Baseline pain intensity, n (\%) }\end{array}$ & $62.03(6.87)$ & $62.44(8.70)$ \\
$\quad$ & $38(66)$ & $39(67)$ \\
$\quad \begin{array}{l}\text { Sederate } \\
\text { Mean duration of surgery }\end{array}$ & $20(34)$ & $19(33)$ \\
(SD), hours & $1.8(0.43)$ & $1.8(0.51)$ \\
Mean duration of anesthesia & $2.5(0.47)$ & $2.4(0.55)$ \\
(SD), hours & & \\
\hline
\end{tabular}

cantly greater in the parecoxib group compared with placebo beginning at 1.5 hours postdose (1.63 [0.85] vs 1.07 [0.90], respectively; $p=0.01$ ) and for each subsequent time point throughout the 24-hour evaluation period ( 1.35 [1.31] vs 0.61 [0.80], respectively, at 24 hours; $p \leq 0.001$ for all time points; Figure 2B). Mean (SD) PRID was significantly greater in the parecoxib group compared with placebo starting at 1 hour postdose (2.04 [1.33] vs 1.46 [1.36], respectively; $p<0.05)$ and for each subsequent time point throughout the 24-hour evaluation period (1.89 [2.05] vs 0.55 [1.46], respectively, at 24 hours; $p<0.001$ for all time points; Figure 2C). Median time to rescue medication (hours:minutes) was significantly longer with parecoxib $(21: 30$ [95\% CI, 13:30 to >24:00] compared with placebo (4:08 [95\% CI, 3:03-6:50]; $p<0.001$; Figure 2D). Additionally, 28 of $54(52 \%)$ patients in the parecoxib group took rescue medication compared with 51 of $57(89 \%)$ patients in the placebo group $(p<0.001)$.

Mean (SD) PPID was significantly greater in the parecoxib group (1.2 [0.7]) compared with placebo (0.8 [0.7]; $p<0.001$; Figure 3A). Mean (SD) PPR was also significantly greater in the parecoxib treatment group compared with placebo (2.6 [0.8] vs 1.6 [0.9], respectively; $p<0.001$; Figure 3B). A significantly greater proportion of parecoxib-treated patients $(70 \%)$ rated study medication as good or excellent compared

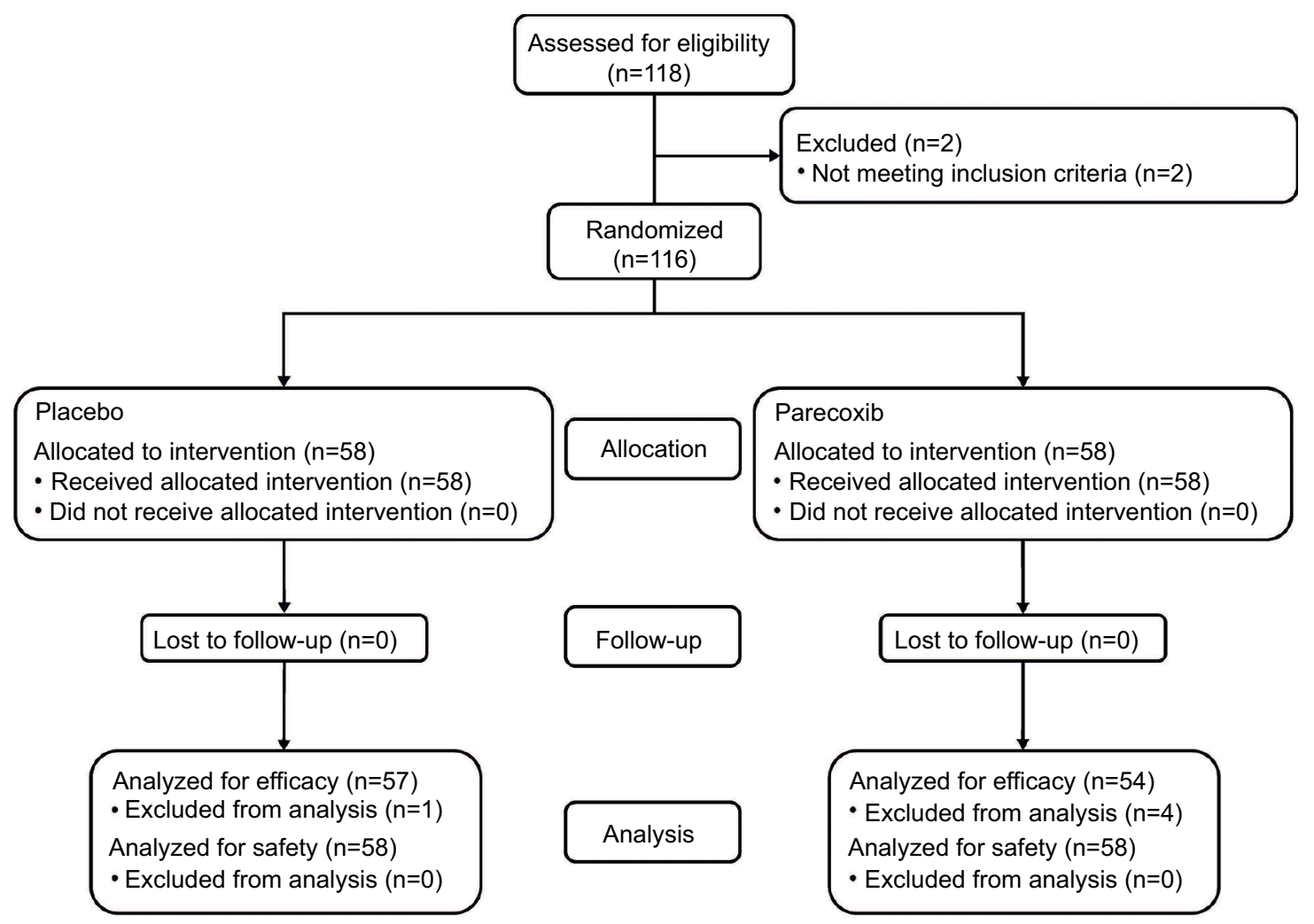

Figure I Patient disposition. 
A

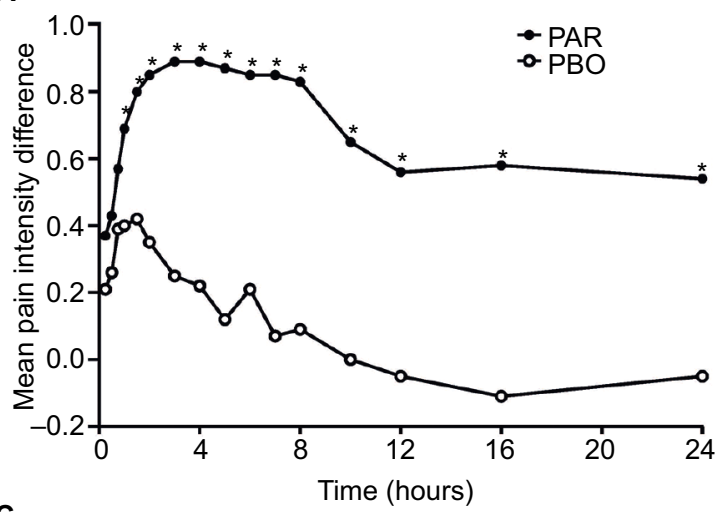

C

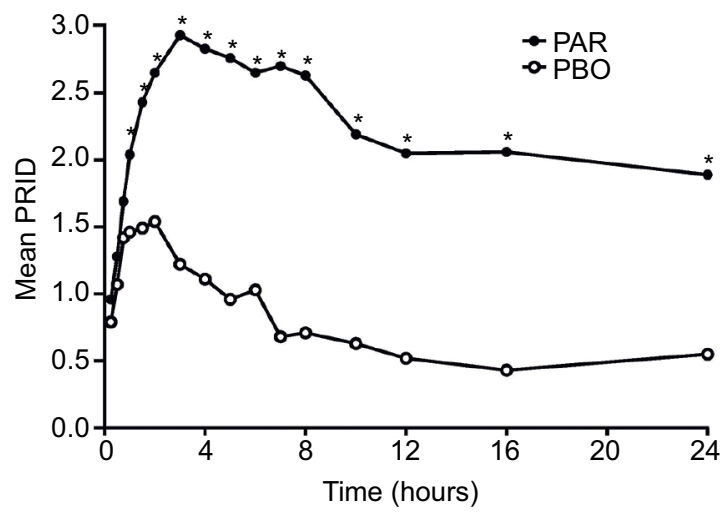

B

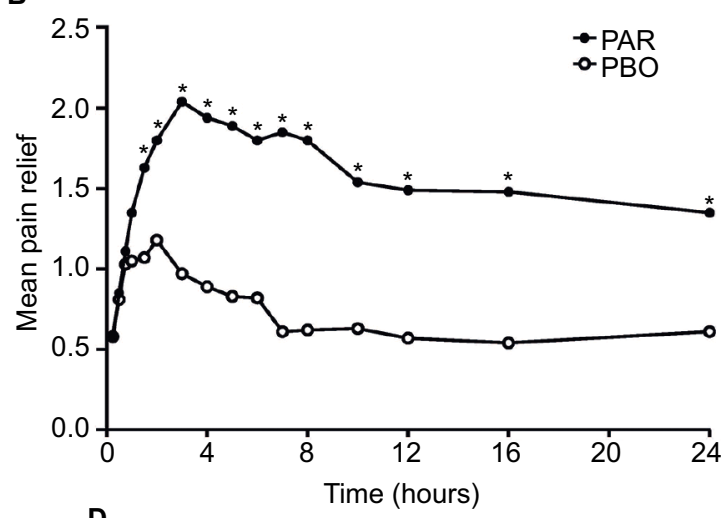

D

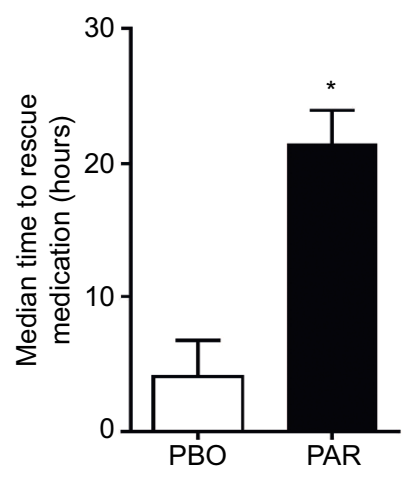

Figure 2 Pain intensity difference (A), pain relief (B), time-specific sum of PID and PR (C), and median time to rescue medication (D) for parecoxib vs placebo. Notes: (A-C) Data shown are means. For these measurements, a larger number indicates greater pain relief improvement. (D) Data shown are median with upper $95 \%$ Cl. ${ }^{*} p \leq 0.05$.

Abbreviations: PAR, parecoxib; PBO, placebo; PID, pain intensity difference; PR, pain relief; PRID, sum of PID and PR.

A

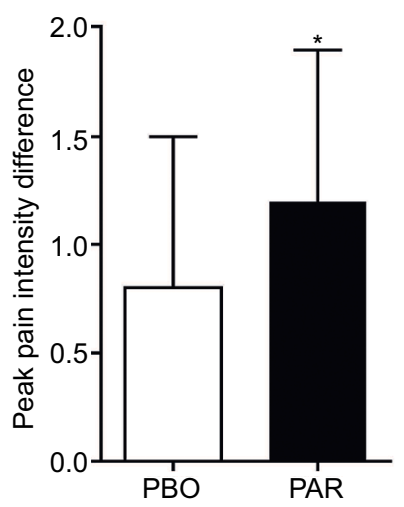

B

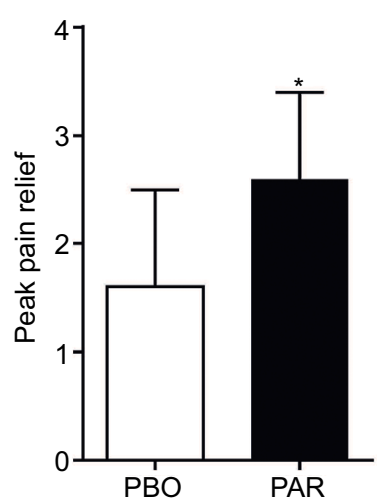

Figure 3 Peak pain intensity difference $(\mathbf{A})$ and peak pain relief $(\mathbf{B})$.

Note: Data are mean $\pm S D$. ${ }^{*} p \leq 0.001$.

Abbreviations: PAR, parecoxib; PBO, placebo.

with placebo-treated patients $(24 \% ; p<0.001)$. Thirty percent of parecoxib-treated patients rated the study medication as poor or fair, compared with $76 \%$ of placebo-treated patients.

\section{Safety}

Thirty-four patients reported treatment-emergent AEs: 22 (37.9\%) patients in the placebo group and 12 (20.7\%) patients

in the parecoxib group. Common treatment-emergent AEs are shown in Table 2. Generally, fewer AEs were reported in the parecoxib group compared with placebo. Significantly fewer parecoxib-treated patients reported fever compared with placebo $(p<0.05)$. AEs were generally mild to moderate in severity and none caused discontinuation from the study. One incidence of pruritus in the parecoxib-treatment group was considered severe by the investigator, but resolved within 2 hours and was not considered related to study treatment. The investigator's opinion on the suspected cause of this severe AE was not captured as part of the trial. There were two AEs in the parecoxib group that were deemed treatmentrelated by the investigator (pruritus; $n=2$ ). No serious AEs or deaths were reported.

\section{Discussion}

Parecoxib is an efficacious, well-tolerated postoperative pain treatment that is approved in over 80 countries, and positive clinical studies have been conducted in various countries, such as the USA, ${ }^{11,12,23}$ France, ${ }^{24}$ Belgium, France, Germany, and Sweden. ${ }^{25}$ The present study showed that a single IV dose of $40 \mathrm{mg}$ parecoxib provided significant analgesia for 
Table 2 Treatment-emergent AEs occurring in $\geq 5 \%$ of either treatment group

\begin{tabular}{llll}
\hline $\mathbf{A E}$ & $\begin{array}{l}\text { Placebo } \\
\mathbf{n = 5 8}\end{array}$ & $\begin{array}{l}\text { Parecoxib } \\
\mathbf{n = 5 8}\end{array}$ & $\mathbf{p}$-value \\
\hline $\mathrm{n}(\%)$ & $22(37.9)$ & $12(20.7)$ & $\mathrm{NS}$ \\
$\begin{array}{l}\text { Pruritus } \\
\quad \text { Related to study }\end{array}$ & $0(0.0)$ & $4(6.9)$ & $\mathrm{NS}$ \\
$\quad 0(0.0)$ & $2(3.4)$ & $\mathrm{NS}$ \\
$\quad \begin{array}{l}\text { medication } \\
\text { Headache }\end{array}$ & $4(6.9)$ & $2(3.4)$ & $\mathrm{NS}$ \\
$\begin{array}{l}\text { Hypertension } \\
\text { Nausea }\end{array}$ & $4(6.9)$ & $\mathrm{I}(1.7)$ & $\mathrm{NS}$ \\
Fever & $5(8.6)$ & $\mathrm{I}(1.7)$ & $\mathrm{NS}$ \\
\hline
\end{tabular}

Notes: ${ }^{a} \mathrm{AEs}$ were deemed related to study medication in the judgment of the investigator.

Abbreviations: AE, adverse event; NS, not significant.

moderate-to-severe pain in Korean patients after TKA compared with placebo. The median time to rescue medication was also longer in parecoxib-treated patients compared with placebo, and patients rated parecoxib more favorably than did those treated with placebo. These results are strengthened by the fact that nearly one-third of patients reported severe pain at baseline. Overall, few treatment-emergent AEs were reported, most were of mild or moderate severity, and rates were similar between placebo and parecoxib. The AE profile of parecoxib was comparable with previous orthopedic studies. ${ }^{9,11,23,25}$ Statistically fewer patients reported fever with parecoxib treatment compared with placebo, which replicates findings from previous studies. ${ }^{9,23,25}$ The reduced incidence of fever compared with placebo may be due to the anti-inflammatory effects of parecoxib. These data support the use of parecoxib for post-orthopedic surgery pain in a Korean population, a country in which parecoxib is not commercially available. The efficacy and safety findings of this study are consistent with those of other studies that evaluated single-dose IV parecoxib in postoperative pain settings. ${ }^{7,8,10,11}$

The design of the current study replicates a similar study conducted in the USA with the same inclusion and exclusion criteria, type of surgery, postsurgical pain severity (moderate or severe), treatments (parecoxib $40 \mathrm{mg}$ IV and placebo), primary efficacy endpoints, safety evaluation parameters, and analysis plan. ${ }^{11}$ Because the majority (86\%) of patients in the US study were Caucasian, we can directly compare the efficacy and safety of parecoxib between different ethnic populations. Demographic and clinical characteristics at baseline, including pain intensity, were comparable between the different ethnic groups, although there were fewer women in the US study. Similar to Korean patients, Caucasian patients treated with parecoxib had significantly larger PID and PR than placebo as early as 0.5 hours postdose that was maintained throughout the 24-hour evaluation period. The median time to rescue medication (hours:minutes) was significantly longer for parecoxib (5:10) than placebo (1:48). A greater proportion of the parecoxib group $(79 \%)$ rated the study medication as good or excellent compared with $38 \%$ of the placebo group. The US study also found parecoxib to be safe and well tolerated, with most AEs of mild-tomoderate severity. Interestingly, in both studies, pruritus was more common in the parecoxib treatment group than the placebo group, although in neither study was the difference statistically significant. The efficacy and safety of parecoxib are, therefore, comparable between Caucasian and Korean patients, with no major differences apparent. A separate study with a different design ${ }^{25}$ also demonstrated the efficacy and safety of parecoxib $40 \mathrm{mg}$ post-TKA in an almost exclusively (98.5\%) Caucasian European patient population. Parecoxib significantly reduced morphine consumption by $\sim 30 \%$ compared with placebo, and provided significantly more PR and greater treatment satisfaction than placebo.

While the present study suggests that parecoxib is safe and effective for the treatment of pain after knee surgery in a Korean patient population, several study limitations must be considered. The population of this study was nearly all female, which may alter the results because of differences in baseline pain perception between men and women. ${ }^{26,27}$ Additionally, the present study may be difficult to replicate, as parecoxib is not commercially available in Korea. Finally, the 4-point categorical pain scale used in this study differs from the more widely utilized 11-point visual analog scale.

\section{Conclusion}

The results of this study demonstrate that single-dose, $40-\mathrm{mg}$ IV parecoxib provides significant analgesia compared with placebo and is well tolerated in Korean patients following unilateral TKA. These results are comparable to those from clinical studies in Caucasian patients, indicating comparable efficacy and safety between Korean and Caucasian patients.

\section{Acknowledgments}

Medical writing support was provided by Shannon Buckley, $\mathrm{PhD}$, and David Cope, $\mathrm{PhD}$, of Engage Scientific Solutions and was funded by Pfizer. This study was sponsored by Pfizer.

\section{Disclosure}

All authors of this study are full-time employees of, and hold stock in, Pfizer Inc. The authors report no other conflicts of interest in this work. 


\section{References}

1. Koh IJ, Kim TK, Chang CB, Cho HJ, In Y. Trends in use of total knee arthroplasty in Korea from 2001 to 2010. Clin Orthop Related Res. 2013;471(5):1441-1450.

2. Maheshwari AV, Blum YC, Shekhar L, Ranawat AS, Ranawat CS. Multimodal pain management after total hip and knee arthroplasty at the Ranawat orthopaedic center. Clin Orthop Relat Res. 2009;467(6):1418-1423.

3. Perkins FM, Kehlet H. Chronic pain as an outcome of surgery. A review of predictive factors. Anesthesiology. 2000;93(4):1123-1133.

4. Coderre TJ, Katz J, Vaccarino AL, Melzack R. Contribution of central neuroplasticity to pathological pain: review of clinical and experimental evidence. Pain. 1993;52(3):259-285.

5. Garimella V, Cellini C. Postoperative pain control. Clin Colon Rectal Surg. 2013;26(3):191-196.

6. Korean Knee Society. Guidelines for the management of postoperative pain after total knee arthroplasty. Knee Surg Related Res. 2012;24(4):201-207.

7. Barton SF, Langeland FF, Snabes MC, et al. Efficacy and safety of intravenous parecoxib sodium in relieving acute postoperative pain following gynecologic laparotomy surgery. Anesthesiology. 2002;97(2): 306-314.

8. Mehlisch DR, Desjardins PJ, Daniels S, Hubbard RC. Single doses of parecoxib sodium intravenously are as effective as ketorolac in reducing pain after oral surgery. J Oral Maxillofac Surg. 2003;61(9): 1030-1037.

9. Viscusi ER, Gimbel JS, Halder AM, Snabes M, Brown MT, Verburg KM. A multiple-day regimen of parecoxib sodium $20 \mathrm{mg}$ twice daily provides pain relief after total hip arthroplasty. Anesth Analg. 2008;107(2):652-660.

10. Ittichaikulthol W, Prachanpanich N, Kositchaiwat C, Intapan T. The post-operative analgesic efficacy of celecoxib compared with placebo and parecoxib after total hip or knee arthroplasty. J Med Assoc Thai. 2010;93(8):937-942.

11. Rasmussen GL, Steckner K, Hogue C, Torri S, Hubbard RC. Intravenous parecoxib sodium for acute pain after orthopedic knee surgery. Am J Orthop (Belle Mead NJ). 2002;31(6):336-343.

12. Tang J, Li S, White PF, et al. Effect of parecoxib, a novel intravenous cyclooxygenase type- 2 inhibitor, on the postoperative opioid requirement and quality of pain control. Anesthesiology. 2002;96(6):1305-1309.
13. Schafer AI. Effects of nonsteroidal antiinflammatory drugs on platelet function and systemic hemostasis. J Clin Pharmacol. 1995;35(3):209-219.

14. Laine L, Takeuchi K, Tarnawski A. Gastric mucosal defense and cytoprotection: bench to bedside. Gastroenterology. 2008;135(1):41-60.

15. Campbell CM, Edwards RR. Ethnic differences in pain and pain management. Pain Manag. 2012;2(3):219-230.

16. Fabian LA, McGuire L, Goodin BR, Edwards RR. Ethnicity, catastrophizing, and qualities of the pain experience. Pain Med. 2011;12(2): 314-321.

17. Rowell LN, Mechlin B, Ji E, Addamo M, Girdler SS. Asians differ from non-Hispanic Whites in experimental pain sensitivity. Eur J Pain. 2011;15(7):764-771.

18. Rabow MW, Dibble SL. Ethnic differences in pain among outpatients with terminal and end-stage chronic illness. Pain Med. 2005;6(3):235-241.

19. Merry B, Campbell CM, Buenaver LF, et al. Ethnic group differences in the outcomes of multidisciplinary pain treatment. $J$ Musculoskelet Pain. 2011;19(1):24-30.

20. Dominick KL, Bosworth HB, Hsieh JB, Moser BK. Racial differences in analgesic/anti-inflammatory medication use and perceptions of efficacy. J Natl Med Assoc. 2004;96(7):928-932.

21. Miller RG. Survival Analysis. NY, USA: John Wiley and Sons, Inc.; 1981:74-75.

22. Simon R, Lee YJ. Nonparametric confidence limits for survival probabilities and median survival time. Cancer Treat Rep. 1982;66(1):37-42.

23. Malan TP Jr, Marsh G, Hakki SI, Grossman E, Traylor L, Hubbard RC. Parecoxib sodium, a parenteral cyclooxygenase 2 selective inhibitor, improves morphine analgesia and is opioid-sparing following total hip arthroplasty. Anesthesiology. 2003;98(4):950-956.

24. Martinez V, Belbachir A, Jaber A, et al. The influence of timing of administration on the analgesic efficacy of parecoxib in orthopedic surgery. Anesth Analg. 2007;104(6):1521-1527.

25. Hubbard RC, Naumann TM, Traylor L, Dhadda S. Parecoxib sodium has opioid-sparing effects in patients undergoing total knee arthroplasty under spinal anaesthesia. Br J Anaesth. 2003;90(2):166-172.

26. Chesterton LS, Barlas P, Foster NE, Baxter GD, Wright CC. Gender differences in pressure pain threshold in healthy humans. Pain. 2003;101(3):259-266.

27. Bartley EJ, Fillingim RB. Sex differences in pain: a brief review of clinical and experimental findings. Br J Anaesth. 2013;111(1):52-58.
Journal of Pain Research

\section{Publish your work in this journal}

The Journal of Pain Research is an international, peer reviewed, open access, online journal that welcomes laboratory and clinical findings in the fields of pain research and the prevention and management of pain. Original research, reviews, symposium reports, hypothesis formation and commentaries are all considered for publication.

\section{Dovepress}

The manuscript management system is completely online and includes a very quick and fair peer-review system, which is all easy to use. Visit http://www.dovepress.com/testimonials.php to read real quotes from published authors. 